\title{
Fuzzy-Neuropsychiatric Predictive Model
}

\author{
Amadin, F. I ${ }^{1}$ and Obi, J.C. ${ }^{2}$ \\ Department of Computer Science, University of Benin, Benin City. Nigeria \\ frankamadin@uniben.edu ${ }^{1}$ and tripplejo2k2@yahoo.com²
}

\begin{abstract}
Due to subjective diagnosis approach, psychiatric diagnosis, prognosis and predication has been tremendously difficult. In short, it has been subjective; tied to a particular human expert with overview on psychiatric diagnosis. Overtimes, inadequate, ineffective and exhaustive medical professional has contributed seriously to poor psychiatric diagnosis. Artificial Intelligence (AI) has shown rich potential in this area. Therefore this research paper has predictively proposed a fuzzy-neural model capable of imprecision or vague handling in collaboration with system self-learning or training using fuzzy logic and neural, subdomain of Soft-Computing (SC) and Artificial intelligence. The various views of the model were visualized using Unified Modeling Language (UML) while system implementation was opened to future research.
\end{abstract}

Keyword: Fuzzy Logic, Neural Network, Psychiatric, UML

\section{Introduction}

Mental and behavioral disorders, which results to mental illness have been a major focus by the World Health Organization (WHO, 2004,). Hundreds of millions of people worldwide are affected by mental disorders (Mathers and Lancer 2006). WHO estimates that 154 million people suffer from depression and 25 million people from schizophrenia; 91 million people are affected by Alcohol use disorders and 15 million by drug use disorders (WHO, 2004). As many as 50 million people suffer from epilepsy and 24 million from Alzheimer and other dementias (WHO, 2004). Around 877000 people die by suicide every year3. Within countries, the overall one-year prevalence ranges from $4 \%$ to $26 \%$ (FMOH, 2004 and WHO, 2004). In high-income countries, depression a lone is likely to be the second highest cause of mental disorders contributing to the overall disease burden.

In Africa and Nigeria as a whole the rate of mental disorder is seriously alarming, this has resulted to serious issues such as stigmatization, disdainful approach and outright insults (FMOH, 2004).

The issues relating to mental health symptoms and correct diagnoses are contained in the International Classification of Mental and Behavioral Disorders (ICD-10) as well as in the Diagnostic Criteria for Research (DCR-10). The successful treatment and interventions require appropriate diagnosis. A major issue faced by psychiatrist is proper diagnosis since symptoms of many mental and behavioral disorders are similar (WHO, 2004).

The fuzzy nature pertaining to the symptoms of varied mental disorder has indeed hinder objective diagnosis and prognosis, leaving room for inadequate or failed diagnosis. Proposing a Neuro-fuzzy, DOI: 10.14738/jbemi.22.1036 
Kanika Sharma and Deepti Mittal; Fuzzy-Neuropsychiatric Predictive Model. Journal of Biomedical Engineering and Medical Imaging, Volume 2, No 2, April (2015) , pp 14-21

Neuro-psychiatic system capable of providing an objective diagnosis cannot be over emphasized which is the centered thrust of this research paper.

\section{Review of Related Literature}

Neural network (NN) consists of an interconnected group of neurons (Ponniyin, 2009). Artificial Neural Network (ANN) is made up of interconnecting artificial neurons (Programming constructs that mimic the properties of biological neurons). A Neural Network is an analog and parallel computing system. A neural network is made up of a number of very simple processing elements that communicate through a rich set of interconnections with variable weights or strength. ANN (subsequently referred to as NN) is used in solving artificial intelligence problems without creating a model of a real biological system. NN processes information using connectionist approach to computation. It changes it structures based on internal or external information that flows through the network during the learning phase. NN can be used to model complex relationship between input and output or find patterns in data (Gary and George, 2002).

Figure 1 presents a simple NN which comprises of three layers (Input, Hidden and Output layers).

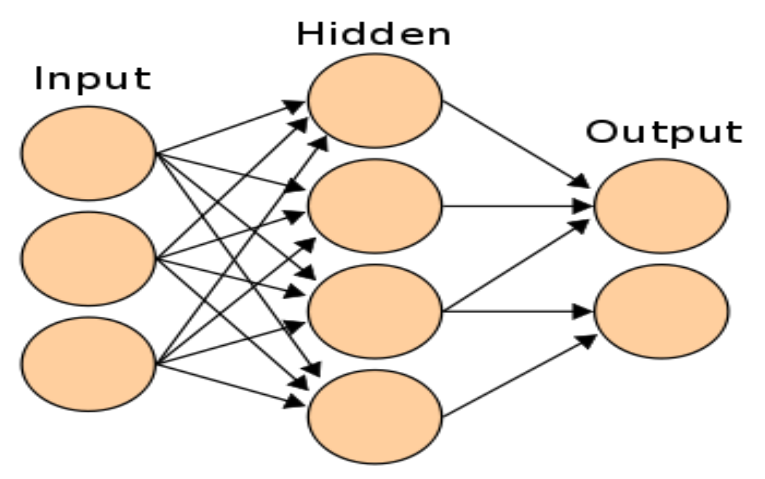

Figure 1: A simple Neural Network (Gary and George, 2002).

The NN presented in Figure 1, comprises of a layer of "input" connected to a layer of "hidden" units, which is in turn connected to a layer of "output" units. The activity of the input unit represents the raw information that is fed into the network; the activity of the hidden units is determined by the activity of the input unit and the weights between the hidden and output units. The hidden units are free to construct their own representation of the input; the weights between the input and hidden units determine when each hidden unit is active, and so by modifying these weights, a hidden unit can choose what it represents (Christos and Dimitros, 2008).

NN employs learning paradigm that includes supervised, unsupervised and reinforcement learning (Christos and Dimitros, 2008). NN has been applied in stock market prediction, credit assignment, monitoring the condition of machinery and medical diagnosis (Dase and Pawar, 2010; Hiroshi et al. 2011; Adyles and Fabrício, 2010; Vahid and Gholam, 2009). Application of NN in medical diagnosis includes electronic noses and diagnosis of cardiovascular systems (Jionghua et al, 2010). NN are ideal in recognizing diseases using scans. They learn by example, hence details of how to recognize the disease is not needed. What is needed is set of examples that are representatives of all the variation of the disease. However, NN cannot handle linguistic information and also cannot manage imprecise or vague information (Jionghua et al, 2010). 
Fuzzy Logic (FL) helps computers paint vivid pictures of the uncertain world. Fuzzy sets were introduced by Zadeh (1965) as a means of representing and manipulating data that are not precise, but rather fuzzy. Fuzzy logic provides an inference morphology that helps appropriate human reasoning capabilities to be applied to knowledge-based systems. The theory of fuzzy logic provides a mathematical strength to capture the uncertainties associated with human cognitive processes, such as thinking and reasoning. A fuzzy set $A$ is called trapezoidal fuzzy number (Figure 2) with tolerance interval [a, b], left width $\alpha$ and right width $\beta$ if its membership function has the following form

$$
A(t)= \begin{cases}1-(a-t) / \alpha & \text { if } a-\alpha \leq t \leq a \\ 1 & \text { if } a \leq t \leq b \\ 1-(t-b) / \beta & \text { if } a \leq t \leq b+\beta \\ 0 & \text { otherwise }\end{cases}
$$

and we use the notation $A=(a, b, \alpha, \beta)$. It can easily be shown that $[A]^{\gamma}=[a-(1-\gamma) \alpha, b+(1-\gamma) \beta], \forall \gamma \varepsilon$ $[0,1]$. The support of $A$ is $(a-\alpha, b+\beta)$.

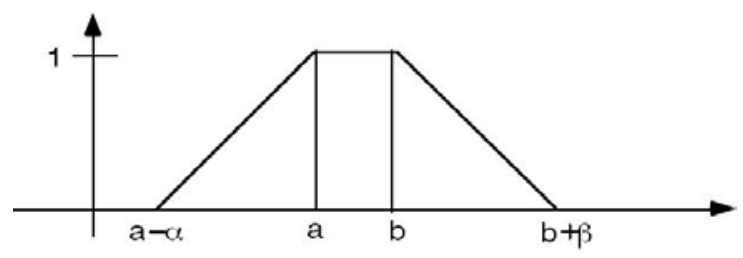

Figure 2: Trapezoidal fuzzy number (Leondes, 2010)

Expert systems are knowledge-based systems that contain expert knowledge. An expert system is a program that can provide expertise for solving problems in a defined application area in the way the experts do. They use human knowledge to solve problems that normally would require human intelligence. These expert systems represent the expertise knowledge as data or rules within the computer. These rules and data can be called upon when needed to solve problems (PCAI, 2002; NIJ 2011 and Steffen 2011).

Fuzzy systems often learn their rules from experts. When no expert gives the rules, adaptive fuzzy systems learns by observing how people regulate real systems (Leondes, 2010). The difference between classical and fuzzy logic is something called "the law of excluded middle" (Bart and Satoru, 1993 and Ahmad, 2011). In standard set theory, an object does or does not belong to a set. There is no middle ground. In such bivalent systems, an object cannot belong to both its set and its compliment set or to neither of them. This principle preserves the structure of the logic and avoids the contradiction of object that both is and is not a thing at the same time (Zadeh 1965). However, fuzzy logic is highly abstract and employs heuristic (experiment) requiring human experts to discover rules about data relationship (Angel and Rocio, 2011).

Fuzzy Neural Network or Neuro-Fuzzy system is a learning machine that finds the parameters of a fuzzy system (i.e., fuzzy sets, fuzzy rules) by exploiting approximation techniques from neural networks (Statsoft Incorporated, 2008). Neuro-fuzzy refers to the combination of artificial neural network and fuzzy logic. It eliminates the individual weaknesses of neural network and fuzzy logic while making use of their best advantages. Fusion of neural network and fuzzy logic (that is Neuro-fuzzy) is interesting 
Kanika Sharma and Deepti Mittal; Fuzzy-Neuropsychiatric Predictive Model. Journal of Biomedical Engineering and Medical Imaging, Volume 2, No 2, April (2015) , pp 14-21

(Jionghua et al, 2010; Saman, 2010; Stathacopoulou et al., 2004). Neuro-fuzzy system for the diagnosis of hypotension will provide a self-learning and adaptive system that is able to handle uncertain and imprecise data.

\section{Methodology and Design of the Proposed Fuzzy-Neuropsychiatric Predictive Model}

The methodology was centered on proposing a Fuzzy-Neuropsychiatric predictive model, which is an architectural framework which enhances the fuzzy (inexact) psychiatric symptoms with the aim of establishing a conclusive boundary point. Unlike the current approaches, in which success or failure are based on the wills and experiences of relevant psychiatric expert personnel designing and administrating the approach in other to elicit relevant diagnosis points. This model is artificial intelligence based; therefore success and failure are not dependent on human intuitions, but success, is closely linked within tuned-up variables within the system components. The model is made-up of five main components as Figure 3 clearly depicts:

a. The Input Component (Mental Health Symptoms): The mental health symptoms pertaining to an individual patient is inputted into the system.

b. The Processing Fuzzy Membership Component: This component transposes crisp inputted values into fuzzy values for processing optimization.

c. Varied Diagnosis Classes: This is usually inferred from the predefined fuzzy rules, breakdown membership function and the exhibited patient symptoms, which will invariably generate a diagnosis class

d. Supervised Neural Network: Self-learning and training ability.

e. Output: The generated result is transmitted back to the end-user.

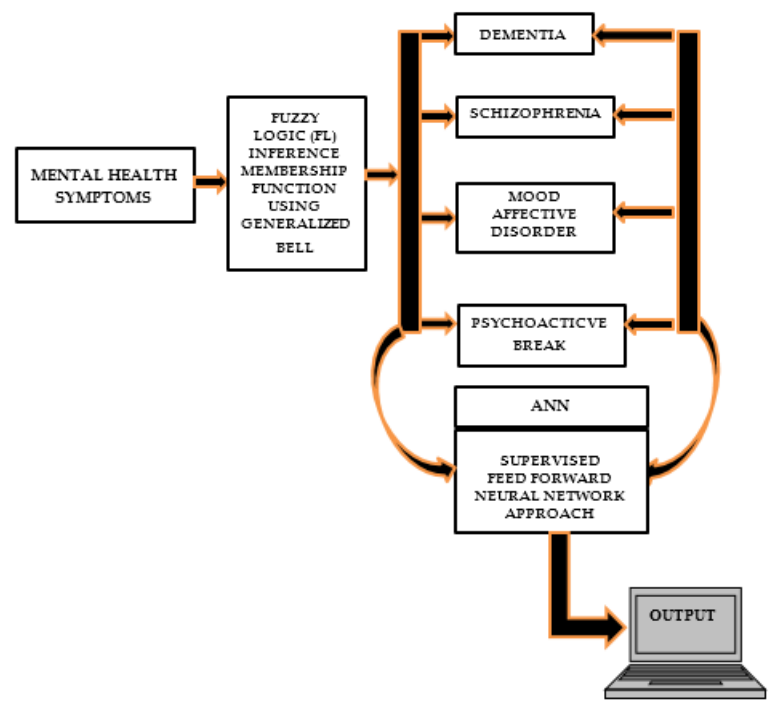

Figure 3: Fuzzy-Neuropsychiatric Predictive Model

\subsection{Design}

Unified modeling language (UML) is a standard modeling language used for modeling software systems. It provides a number of graphical tools that can be used to visualize a system from different viewpoints. 
The multiple views (user, structural, behavior, implementation and environment) of the system that is represented by using diagrams together depict the model of the system (Philippe, 2000 and Chris, 2000). The views typically used are The User view; represents the goal and objectives of the system form user's viewpoint. The structured view; represent the static or idle state of the system. The behavioral view; represents the dynamic or changing aspect of the system. The implementation view; represents the distribution of the logical elements, such as source code structure, runtime implementation structure of the system. The environment view; represents the distribution of the physical elements of the system.

The Unified Modeling Language is used to illustrate the processes involved in the design and implementation of the model. The model is represented using the following UML diagram specified from Figure 4 -8.

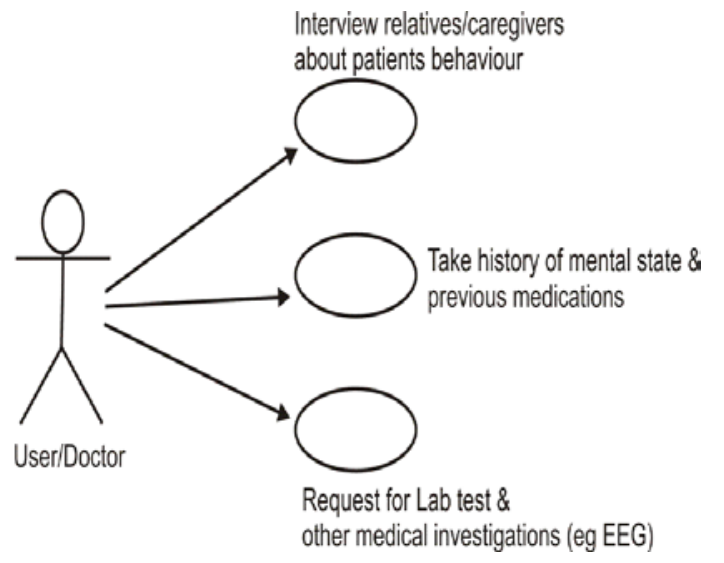

Figure 4: Use-Case Diagram for the Fuzzy-Neuropsychiatric predictive model

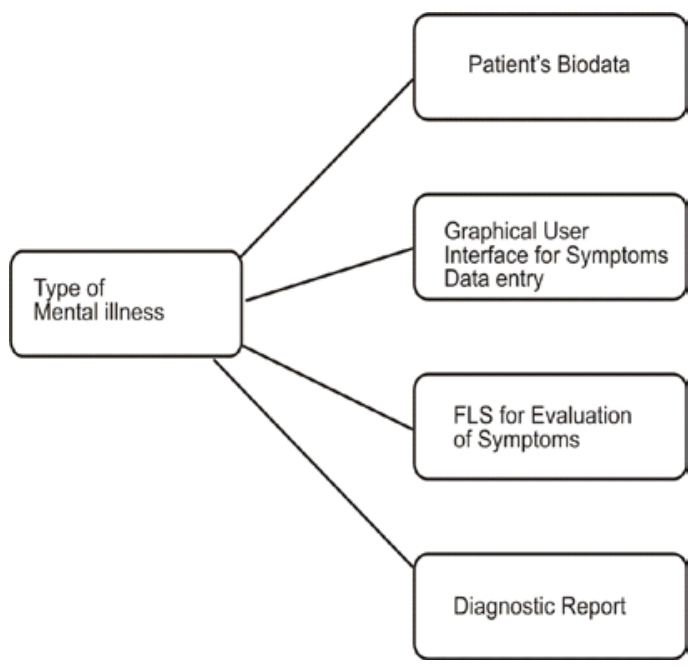

Figure 5: Class Diagram for the Fuzzy-Neuropsychiatric predictive model 
Kanika Sharma and Deepti Mittal; Fuzzy-Neuropsychiatric Predictive Model. Journal of Biomedical Engineering and Medical Imaging, Volume 2, No 2, April (2015) , pp 14-21

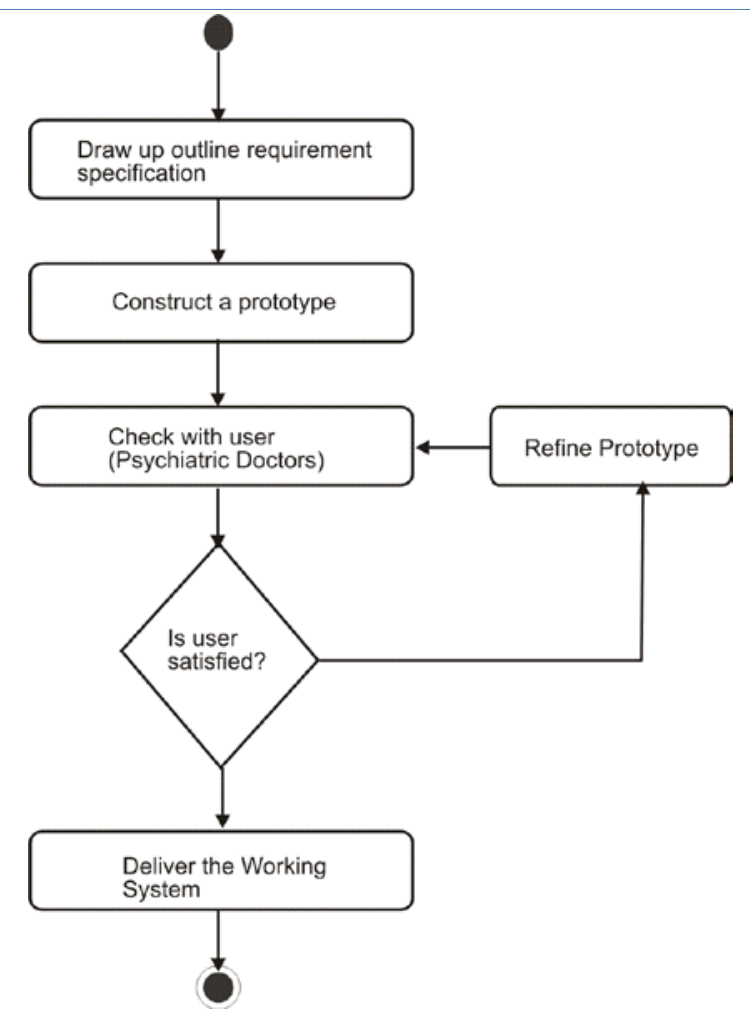

Figure 6: Activity Diagram for the Fuzzy-Neuropsychiatric predictive model

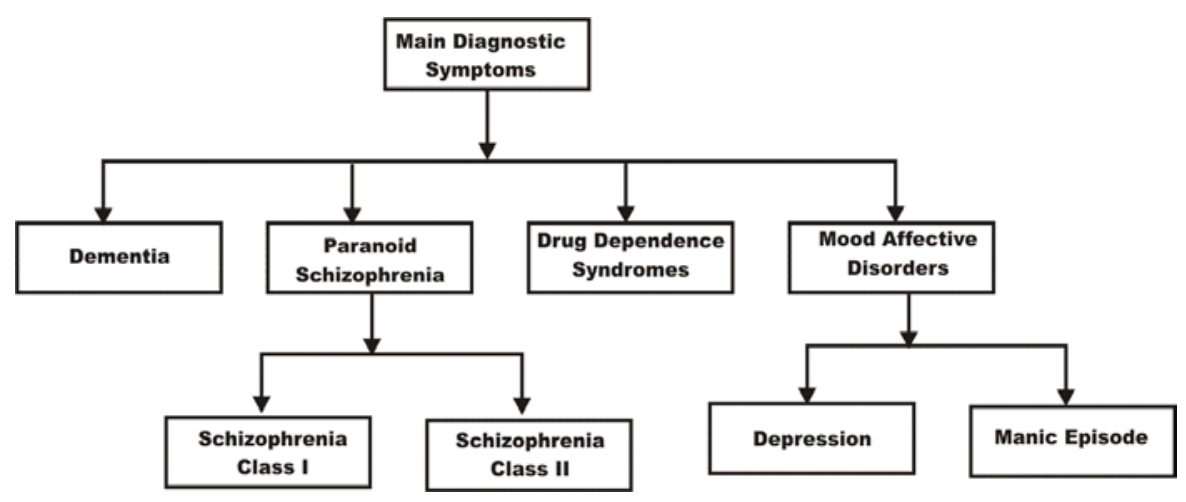

Figure 7: Hierarchy Input-Processing-Output Chart (HIPO) for the Fuzzy-Neuropsychiatric predictive model 


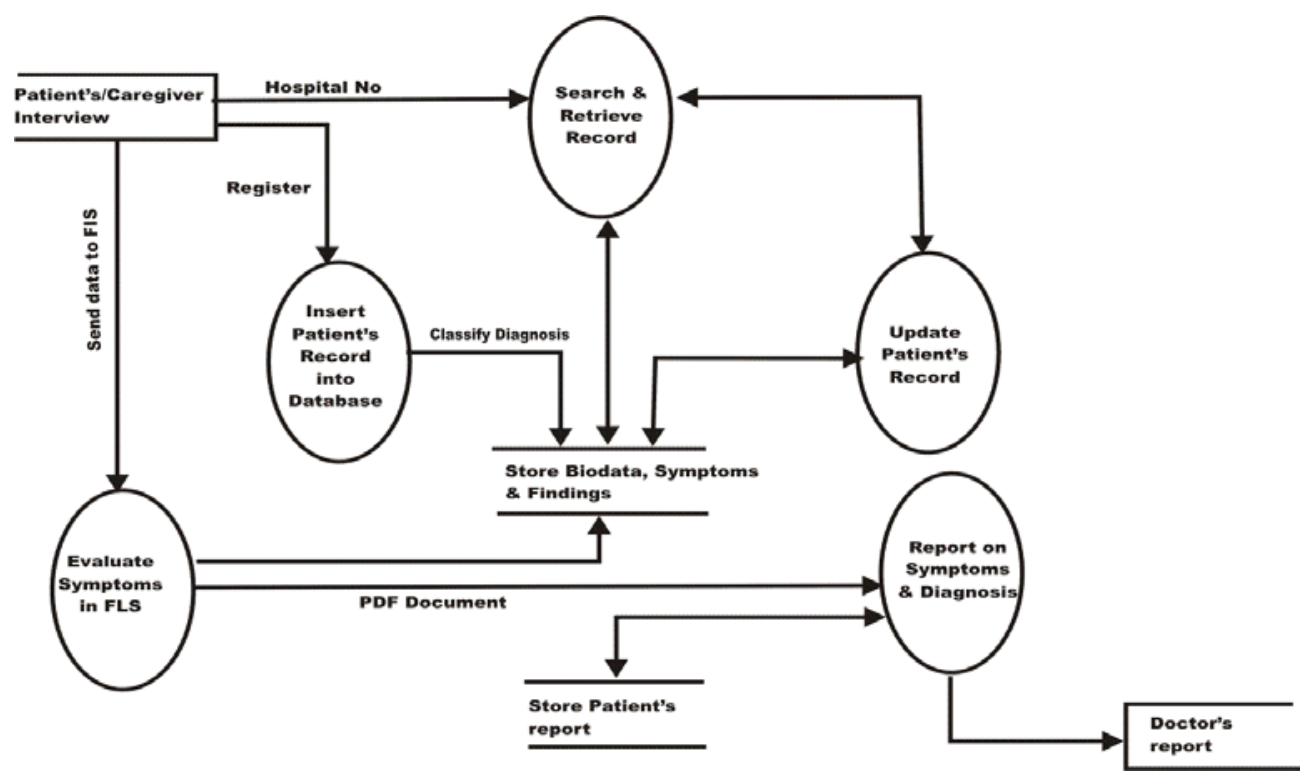

Figure 8: Data Flow Diagram (DFD for the Fuzzy-Neuropsychiatric predictive model

\section{Discussion}

The utilization of UML as a design tool has indeed visualized various aspect of the system from the user view down to the interface and even system implementation phase. This design approach will foster easily system implementation

\section{Conclusions}

A Fuzzy-Neuropsychiatric Predictive Model has been proposed and designed utilizing an informal design tool (UML), highlighting the fuzzy system components and interaction. On full system implementation of the various Artificial Intelligence (Al) techniques, objective diagnosis will have suppressed subject approach, providing a conclusive fuzzy boundary for early, gradual and optimal result.

\section{REFERENCE}

[1] Adyles A. J., Fabrício C. L. A. (2010), “Automatic Faults Diagnosis by Application of Neural Network System and Condition-based Monitoring Using Vibration Signals, retrieved from http://www.informatics.org.cn/doc/ucit201001/ucit20100104.pdf

[2] Angel C. and Rocio R. ( 2011), "Documentation management with Ant colony Optimization Metaheuristic: A Fuzzy Text Clustering Approach Using Pheromone trails" retrieved from soft computing in Industrial applications, Advances in intelligient and soft Computing, 2011 vol. 96, 2011, 261-70, DOI: 10.1007/978-3-642-20505-1_23

[3] Christos S. and Dimitros S. (2008), "Neural Network" retrieved from http//:docs.toc.com/doc/1505/neural-networks.

[4] Dase R.K. and Pawar D.D. (2010), "Application of Neural network to stock market prediction: A review of literature", retrieved from http://www.bioinfo.in/uploadfiles/12843 
Kanika Sharma and Deepti Mittal; Fuzzy-Neuropsychiatric Predictive Model. Journal of Biomedical Engineering and Medical Imaging, Volume 2, No 2, April (2015) , pp 14-21

[5] FMOH (2004), Revised National Health Policy, Federal Ministry of Health, Abuja

[6] Gary R. and George P.E. (2002), "Application of Neuro System to behavior Representation in Computer generated forces", retrieved http//:www.Cuil.com

[7] Hiroshi S.; Kentaro K.; Kazuo O. and Masato O. (2011), "Statistical mechanics of Structural and temporal credit assignment effects on learning in neural Networks" retrieved from http://pre.aps.org/abstract/PRE/v83/i5/e051125.

[8] Jionghua T.; Suhuan W.; Jingzhou Z. and Xue W. (2010), "Neuro-fuzzy logic based fusion algorithm of medical images" retrieved from http:// ieeexplore.ieee.org/xpl./freeabs_all.jsp?

[9] Leondes C. (2010), "The Technology of Fuzzy Logic Algorithm retrieved from Suite101.com/examples-of-expert-System-application-in-artificiallntelligience.

[10] Mathers C.O. and Lancer D. (2006), Projections of global mortality and burden of disease from 2002 to 2030, Plus Medicine, 3: 2011-2030

[11] NIJ: National Institute of Justice (2011), "Expert system technologies for criminal justice application" retrieved from https://www.ncjrs.gov/pdffiles1/nij/sl000959.pdf

[12] PCAI (2000), “Expert System: Introduction, retrieved from http://www. pcai.com/web/ai_info/expert.systems.html.

[13] Ponniyin S.K. (2009), “Neural Network”, Icann2007.org/neural.networks.

[14] Saman K. H. (2010), "Neuro-Fuzzy Systems from the Neural Network Perspective" retrieved from http://citeseerx.ist.psu.edu/viewdoc/summary?doi=10.1.1.39.857

[15] Statsoft Incorporated (2008), "Neural Network" retrieved from http//: google.com.

[16] Steffen L. (2011), "Expert system and local Computation", University of Oxford, Graduate Lectures Hilary Term 2011

[17] Stathacopoulou R.,Magoulas G.D.,Grigoriadou M., and Samarakou M. (2004), "A Neuro-Fuzzy Approach to Detect Student's Motivation, retrieved from http:// et.teiath.gr/English/cv/cv_samarakou.html-

[18] Vahid K. and Gholam A.M. (2009), "Artificial Intelligence in medicines" V47, Issues 1 Information Technology Department, School of Engineering, Terbiat Moderas University Tehran, Iran.

[19] WHO (2004), World Health Report; changing history. Geneva, World Health Organization.

[20] Zadeh L.A. (1965), "Fuzzy sets. Information and control, Vol.8, pp.338-353. 